\title{
Big transfer learning for automated skin cancer classification
}

\author{
Zinah Mohsin Arkah', Dalya S. Al-Dulaimi ${ }^{2}$, Ahlam R. Khekan ${ }^{3}$ \\ ${ }^{1}$ Department of Bioinformatics, University of Information Technology and Communications, Iraq \\ ${ }^{2,3}$ Department of Intelligent Medical System, University of Information Technology and Communications, Iraq
}

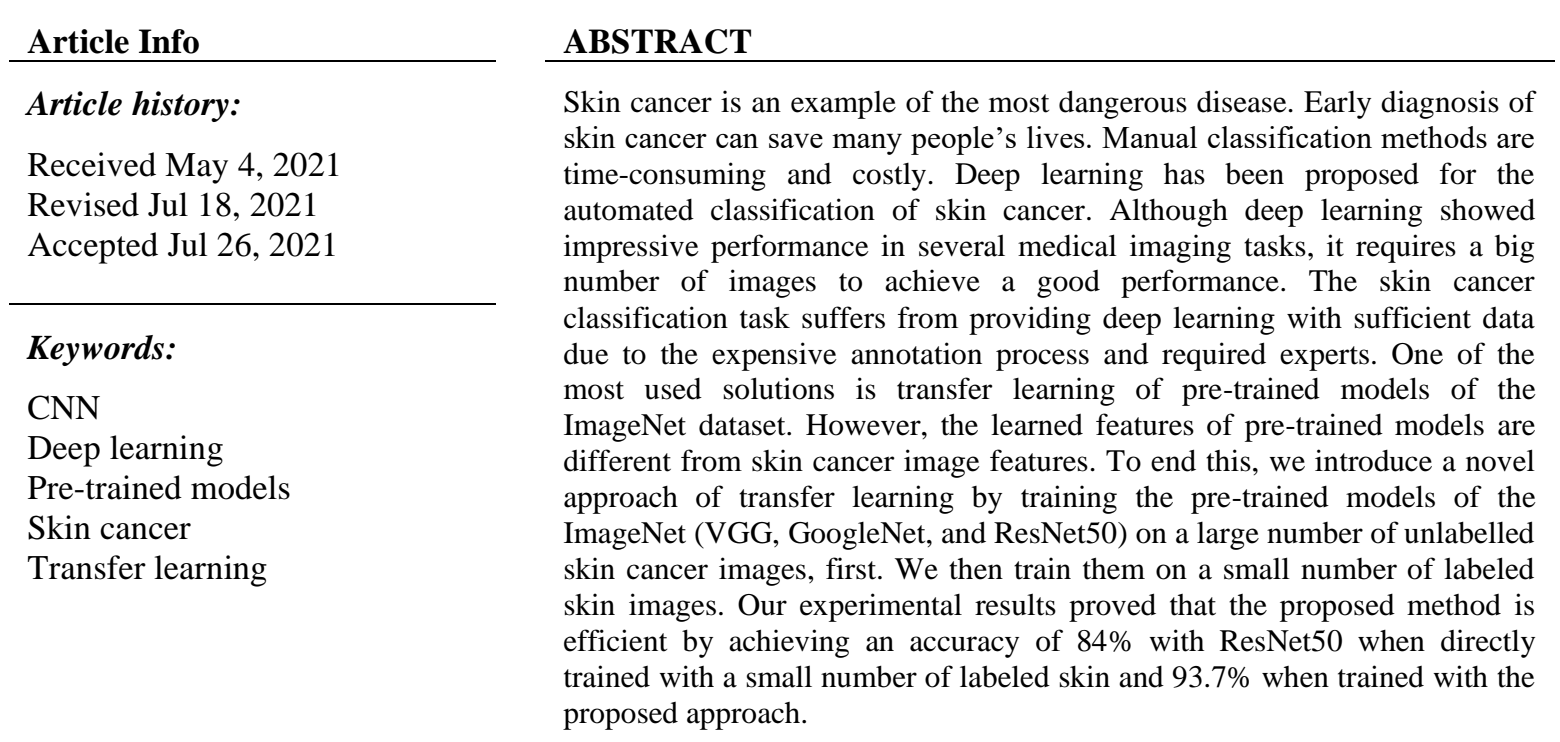

This is an open access article under the CC BY-SA license.

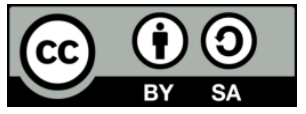

\section{Corresponding Author:}

Zinah Mohsin Arka

Department of Bioinformatics

College of Biomedical Informatics

University of Information Technology and Communications

Baghdad, Iraq

Email: zinah2018@uoitc.edu.iq

\section{INTRODUCTION}

Nowadays, one of the deadliest and fastest-spreading cancers in the world is skin cancer. Seventyfive percent of the skin cancer patients are dying every year [1]-[3], while in the USA, there is a danger of skin cancer in every five persons, mostly living in an extremely high sunshine area and having pale skin type [1]. Also for 2017, more than 87,000 emerging cases of melanoma are expected to diagnose in the USA [2]. In Australia, 1520 people died from melanoma and 642 from non-melanoma in 2015. At early stages of any disease, including skin cancer, can be diagnosed, treated, and cured easily like other diseases, early diagnosis is so significant for handling and can quickly at initial degrees [1]-[4]. For helping dermatologists in clinical testing, a skin cancer diagnosis has been improved by introducing a dermoscopy technique. It is a noninvasive imaging technique which provides a high-quality visual appearance of skin lesion. Dermoscopy is lower screening errors, more satisfactory deep layers details, and fewer surface reflections, as compared with conventional microscopy. Hence, dermoscopy images offer greatly better accuracy and visibility [3]-[5].

Recently, the convolutional neural networks (CNNs) technique, as an example of the deep learning approach, has developed an overpowering existence in image classification tasks [6]-[10]. The exciting and 
brilliant capability of visual representation for detection and recognition tasks, depending on the specified training data group, is the most important advantage of CNN [11]. In general, several research works achieved up-to-date performance in the task of skin cancer classification [12], [13]. The CNN models, pretrained on big datasets of ImageNet [14], provided assuring results for image diagnosis tasks, still exclusive of retraining. Aimed at this explanation, these transferred features are utilized in the analysis of dermoscopic images, as well, in the later years [15], [16]. Note that these features are obtained, by default, from the entirely consistent layers of the CNN formation. The main drawbacks of these deep features are susceptibility or sensitivity to the geometric variants and the scarcity of varieties of local patterns [17]. However, in the case of images having impressive differences in resolution and perspective, it could be a vast difficulty to do analysis immediately utilizing CNN pieces. The generally used solutions are data augmentation and rescaling, such as rotate, flip, or crop [18]. The performance may decrease due to some transformations. For example, the object may not within the background area, or the random crop images might simply pick up an indifferent portion of the gadget in the primary picture. Therefore, the performance enhancement is very restricted and may be worse when employing CNN for medical purposes.

Alternatively, numerous researches applied a combination of local description encoding with deep features techniques for improving the distinction capability of such representations, and to avoid employing direct CNN features as a common image representation [19], [20]. These researches have very high intensive computation because of end-to-end encoding layer integrated with training a CNN structure, the employment of pooling strategy based multiple-scale pyramid to compose FV representations [21], or the sliding-windows adoption to create wide descriptors of defined regions in the primary images [17]. In a later study [22], confined pieces are created arbitrarily of a dermoscopic picture and utilized the patch FV aggregated CNN features for automated melanoma classification. In the previous literature [23], each activated CNN inside the feature map, be able to map out back to an interesting field (a specified area) of the input image and mirror the characteristics of the specified area. As a result, an extra effective and the compacted solution is introduced by [24]. It is founded on compactly collecting local descriptors from a convolutional CNN. Although several solutions (such as data augmentation, pre-trained models of imageNet) were proposed to address the shortage of training data in the task of skin cancer classification, still these solutions are not effective. Therefore, we introduce a new strategy that is dependent on enhancing the learned feature of the pre-trained models by training them on a large number of unlabelled skin cancer images then a small number of labeled skin cancer images as shown in Figure 1. The proposed approach guarantees that the models learning the relevant features and reduce the annotation processing time. Moreover, it can be used with any medical imaging task.

Loading the pre-trained models of ImageNet (VGG, GoogleNet, ResNet50)

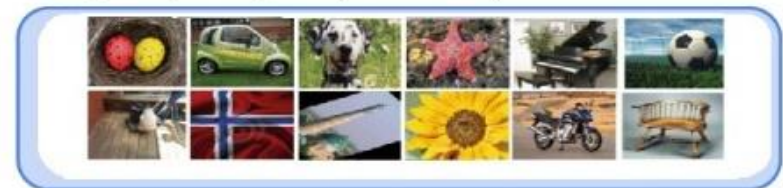

Training the pre-trained models on a large number of unlabeled skin cancer images

Fine-tuning process
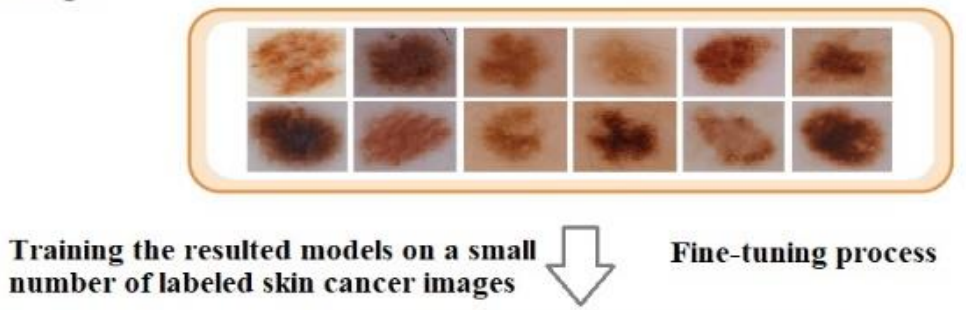
number of labeled skin cancer images

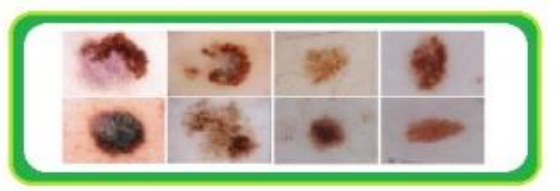

Figure 1. The overall workflow of the proposed method 


\section{RELATED WORK}

The dermoscopy image recognition techniques are categorized into two main categories.

\subsection{Techniques based on the handcrafted feature}

The standard technique for diagnosing colored skin lesions into melanoma and benign is the "ABCD" rule [5]. Several automated diagnosis techniques founded on this rule are proposed [25]. For example, a combination of KNN, feature optimization framework, and hand-designed features (color descriptors, border-gradient, and shape) for differentiation between benign melanoma lesions is adopted by, Ganster et al. [26]. A similar approach has been introduced by Celebi et al. [2] based on extracting a sequence of characteristics from the dermoscopic picture, which includes; texture related descriptors, color, and shape features, and combining them with several algorithms of feature selection to establish a non-linear SVM classifier. Other similar research presented by Capdehourat et al. [27] is based on characterizing each applicant lesion area via descriptor set, which includes texture, color, and shape information, and then employing this information for training the AdaBoost classifier. Moreover, Xie et al. [28] introduced a selfgenerated neural model for generating lesion areas and assembly neural network prototype for feature extraction (border, texture, and tumor color), and then, for melanoma recognition. Bi et al. [29], presented an automated melanoma recognition technique, by utilizing joint reverse classification and multiple scale representations. Considering additional techniques based on obtaining local characteristics (texture and color.) from petite 16x16 patches, and next collected these patches into last representations using the BoF model. In a similar approach, Barata et al. [30], encoded color and texture-related features by applying the BoF model for lesion recognition.

\subsection{Techniques based on deep CNN}

CNN architecture consists of multi-process layers for learning various levels of representations. Therefore, connecting these features maintains very distinguished and efficient deep representations [31][33]. In general, applying CNN for dermoscopy image classification can be categorized in two ways. The first way is the direct-training or fine-tuning extensive model in the end-to-end style. For instance, Demyanov et al. [34] proposed a 5-layer CNN structure for classifying skin lesion data into two different types. Multiple staging systems, founded on the fine-tuning extremely deep remaining system for automatic melanoma classification in dermoscopic pictures, are developed by Yu et al. [35]. In very recent times, Esteva et al. [36] applied a 1-layer extensive model for automated skin cancer recognition. It is founded on GoogleNet Inception V3 architecture and utilized 129450 pictures for training. Another study by Menegola et $a l$, [16] examined the influence of information transference of intense learning in dermoscopy picture classification. The research utilized numerous data sets, such as ImageNet, Retinopathy, ISIC, and Atlas.

The second way is the utilization of deep features extracted from pre-trained CNN in medical image recognition, rather than training the CNN by extremely reliant on calculating resources and large training data. In the analysis field of dermoscopic images, Codella et al, [4] proposed a pre-trained CNN (ImageNet) for discriminating healthy and melanoma images by extracting high-level feature representations. A feature extractor, based on pre-trained CNN by Kawahara et al. [15], is combined with features of sub-image pooling to classify 10-class lesion recognition. Other research by Codella et al. [37], examined the technique called "deep learning ensemble" for melanoma classification. Yu et al. [38] introduced an automated melanoma recognition, via collecting the CNN activations of the randomly selected sub-images from a dermoscopic picture. The main issue with these methods is the shortage of training images [1], [7]. Another issue is the low computational tools. To achieve better results, models should be trained on either GPU [39] or FPGA [40], [41]. In this article, we introduce a novel strategy to address the shortage of training issues and reduce the annotation process time and we train on GPU.

\section{RESEARCH METHOD}

\subsection{Dataset}

In this article, we have utilized two datasets. The first one is ISIC Archive (source dataset) which contains 23,906 dermoscopic images [42]. This dataset is used to train the pre-trained models in the middle stage where huge plenty of unlabeled skin cancer pictures are used. The second dataset is SIIM-ISIC 2020 dataset [43] (target dataset). It contains 33,000 skin lesion pictures divided into two categories: Benign and malignant. It consists of only 584 images of the malignant class and the rest for benign. To have an equal number of images, we took only 584 images from the benign class. 


\subsection{The state-of-the-art architectures}

Numerous CNN architectures, in the area of natural image recognition, are released in recent years, such as VGGNet [44], GoogLeNet [45], and ResNet [46]. A number of these CNNs, like ResNet and GoogLeNet, are offered as pre-trained structures. They are trained on around 1.28 million genetic pictures from the ImageNet database [47]. Hence, the ability to utilize biases and weights for these models, i.e., finetuning all the model layers through continuing with the backpropagation and employing the data, so, the ability to apply them to the specific recognition task, as well. While the VGGNet is initialized so that the biases and weights are not affected by the visual data (might vary from skin images).

The following is a short outline of the generally used CNN architectures:

A. VGGNet: A deep CNN was developed by K. Simonyan, and Zisserman A, [44] in 2015. It consists of extremely small convolutional filters and its depth is in the range of 16-19 layers. It utilized 13 Conv layers with a $3 \times 3$ filter size. Five max-pooling layers are employed to carry out the spatial pooling. Then, several convolutional layers and max-pooling is achieved on a mask of size $2 \times 2$ pixels. Three fully connected layers are following a stack of convolutional layers.

B. GoogLeNet: This type of CNN is presented by Szegedy et al. [45] in 2015. It consists of twenty-two convolutional layers including 9 Inception blocks. Every Inception block has 3 unique filter dimensions, which hold $1 \times 1,3 \times 3$, and $5 \times 5$ for convolutional, as well as, $3 \times 3$ for pooling. Using the given parameters with the RGB color space, the receptive field size is $224 \times 224 \times 3$. In a comparable way to the different CNNs, the preparation step of the convolutional kernel records is dependent on the stochastic gradient descent technique (SGD). The GoogLeNet extracts offline the high-level features of the dissimilar classes through the training phase at the time-demanding, which needs a vast number of training images, as well.

C. The residual network (ResNet): The CNN model with its deep hierarchical architecture has critical significance due to its effective learning ability. He et al. [46], presented a deep residual neural network (ResNet) as a new CNN generation. In the ILSVRC challenge 2016 for feature extraction, which is dedicated to ImageNet large-scale visual recognition, its rank was number one. The key characteristic of ResNet is lying in the addressing capability of the degradation problem while training an extremely deep network (i.e., the adaptation of residual connection), as compared with other classical CNN architectures. As demonstrated earlier, the residual links can preserve the achieved accuracy gains, as well as, accelerate the deep network convergence by considerably enlarging the network depth. In general, when looking inside the deep residual network, it involves a group of residual blocks, with several stacked convolutional layers inside each block. Note that the appendage of the convolutional layer is regarded as the batch normalization layers plus the rectified linear unit layer. The formula of the residual base with connections mapping can be composed as in (1),

$$
h_{l+1}=\operatorname{Relu}\left(h_{l}+F\left(h_{l}, w_{l}\right)\right)
$$

where: $h_{l+1}$ is the n-th residual base output, Relu () is the rectified linear unit function, $h_{l}$ is the n-th residual base input, $\mathrm{F}$ is the residual mapping function, and $w_{l}$ is the block parameters. also note that a linear projection $\varphi$ is normally used for matching the dimensions when $h_{l}$ and $F\left(h_{l}, w_{l}\right)$ are unequal. Next, the (1) can be extra modified as in (2),

$$
h_{l+1}=\operatorname{Relu}\left(\varphi\left(h_{l}\right)+F\left(h_{l}, w_{l}\right)\right)
$$

However, ResNet-101 and ResNet-50 are two different depths of ResNet models. ResNet-50 was utilized in this study which pre-trained on ImageNet.

\subsection{Training procedure and scenarios}

We have trained the pre-trained networks in two different scenarios:

1. Scenario 1: start by adjusting the pre-trained networks then train on the destination dataset.

2. Scenario 2: start by adjusting the pre-trained networks then train on the source dataset as a first step. Figure 2 illustrates the training process. Then adjusting resulted from models from the first step and train on the destination dataset. 


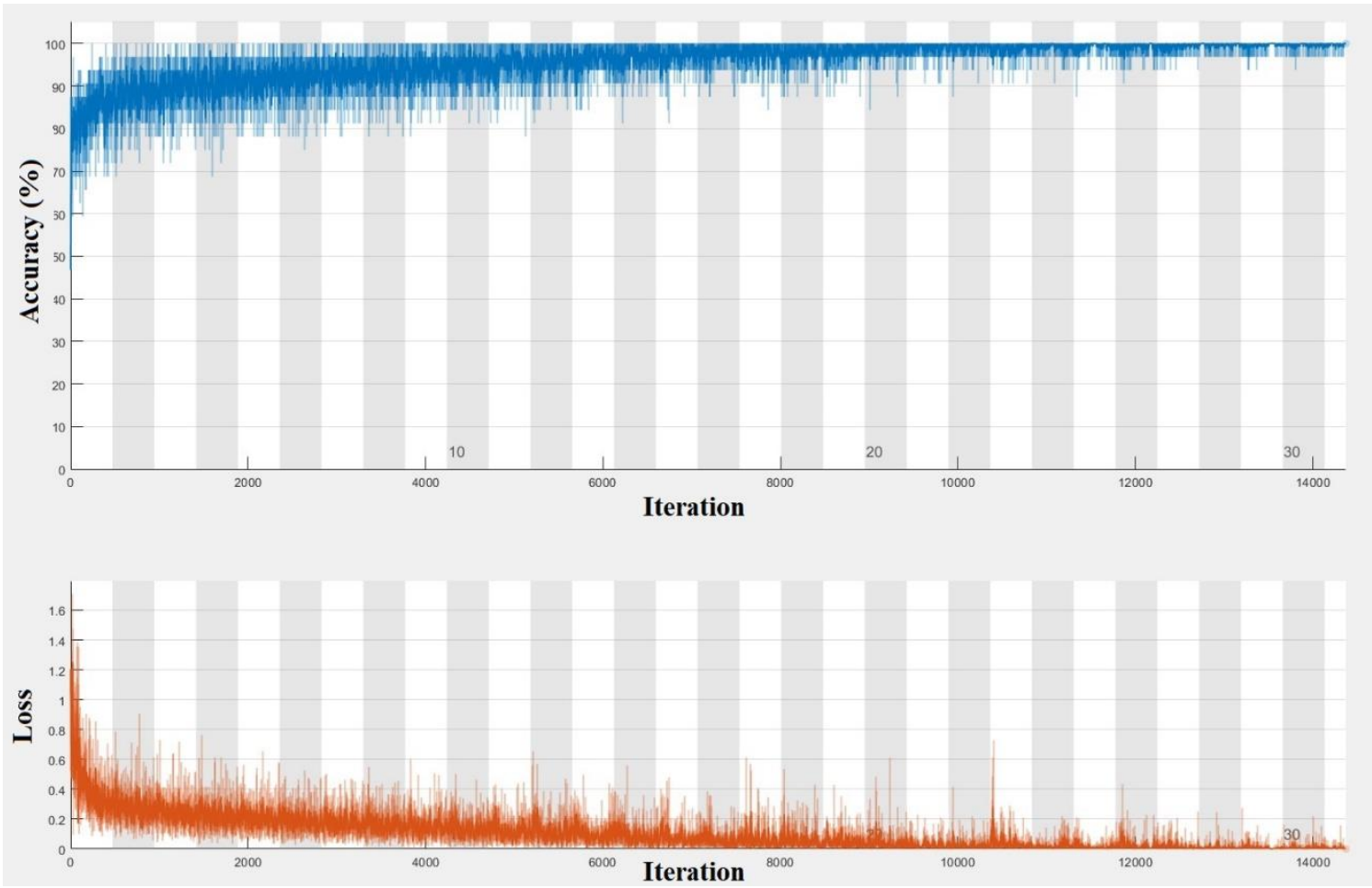

Figure 2. Training process of the first step of scenario 2

We have trained the model for 14000 iterations. Some well-informed kernels from the initial convolution layers of ResNet50 have been shown in Figure 3. The workflow of this scenario is explained in Figure 4.

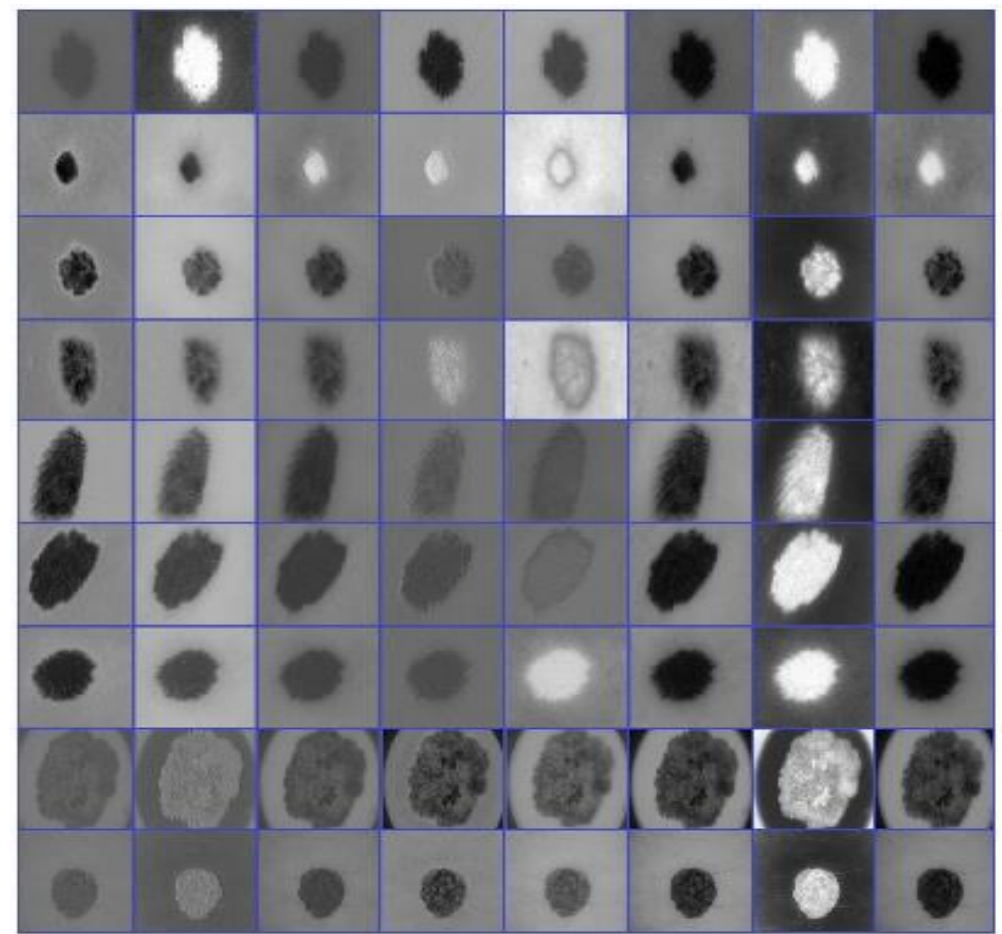

Figure 3. Some learned kernels from the initial convolution layers of ResNet50 

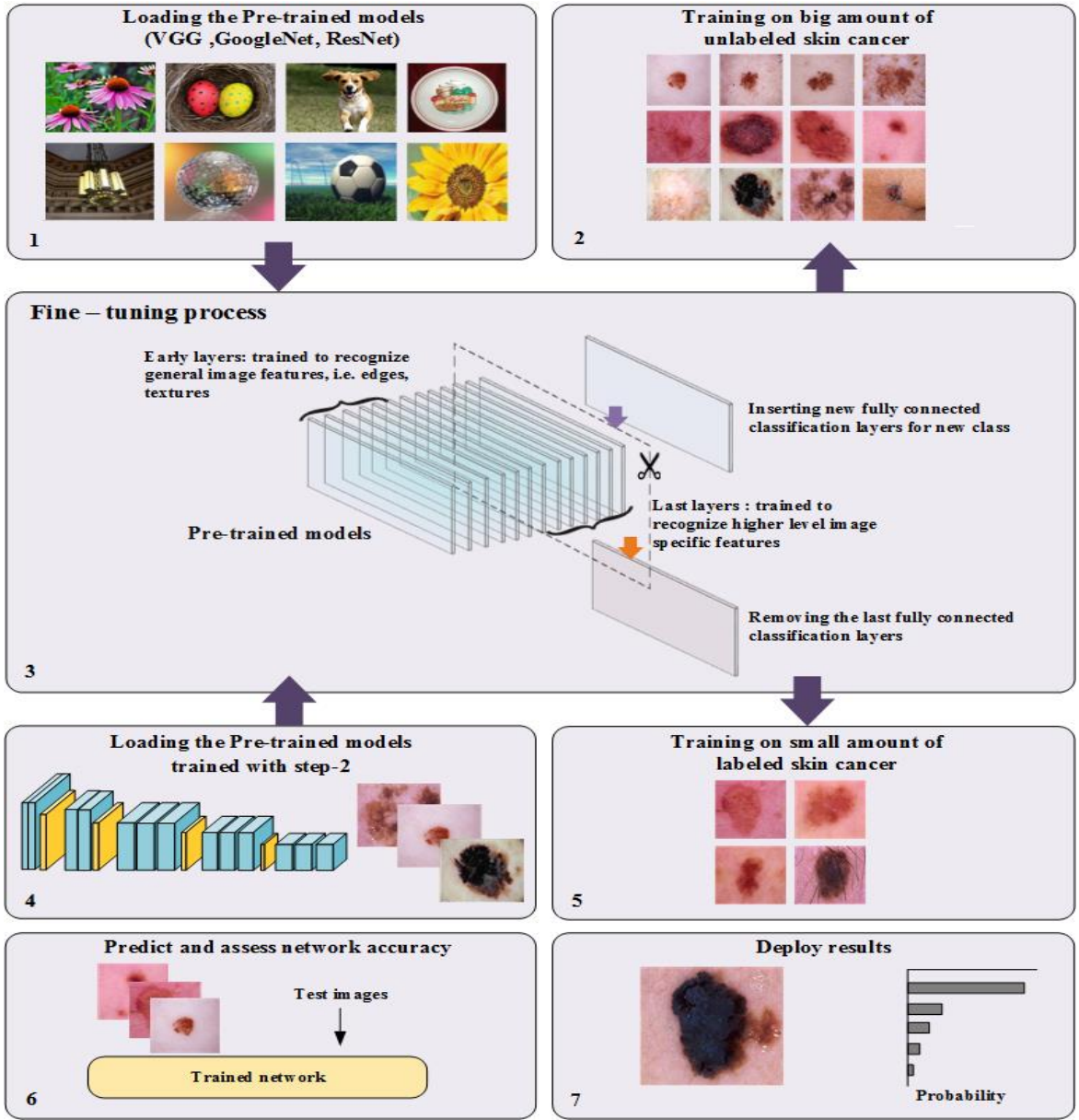

Figure 4. The workflow of scenario 2

\section{EXPERIMENTAL RESULTS}

In this part, we have evaluated the used models on the testing set in several evaluation metrics including accuracy (3), recall (4), precision (5), F1-score (6) [48]-[52].

$$
\begin{aligned}
& \text { Accuracy }=(\mathrm{TP}+\mathrm{TN}) /(\mathrm{TP}+\mathrm{TN}+\mathrm{FP}+\mathrm{FN}) \\
& \text { Recall }=\mathrm{TP} /(\mathrm{TP}+\mathrm{FN}) \\
& \text { Precision }=\mathrm{TP} /(\mathrm{TP}+\mathrm{FP}) \\
& \mathrm{F} 1_{\text {score }}=2 \times((\text { Precision } \times \text { Recall }) /(\text { Precision }+ \text { Recall })
\end{aligned}
$$

We started to evaluate the pre-trained models (VGG19, GoogleNet, ResNet-50) with scenario 1 as reported in Table 1. ResNet 50 is the dominant model among the others by obtaining the highest accuracy, precision, recall, and F1 score of $84.0 \%, 86.6 \%, 89.3 \%, 87.9 \%$ respectively. GoogleNet obtained second place by scoring $81.2 \%$ for accuracy, $84.4 \%$ for precision, $87.5 \%$ for recall, and 85.9 for the F1 score. Lastly, the VGG model achieved the lowest measurements among other models by obtaining $79.6 \%, 83.7 \%$, $85.4 \%, 84.6 \%$ for the accuracy, precision, recall, and F1 score, respectively.

We then evaluated the pre-trained models (VGG19, GoogleNet, ResNet-50) with scenario 2 as detailed in Table 2. The results of all models have been significantly improved. However, the ResNet 50 model is still the dominant model among the others by obtaining the highest accuracy, precision, recall, and F1 score of $93.7 \%, 95.7 \%, 94.6 \%, 95.1 \%$ respectively. GoogleNet earned second place by obtaining 88.8 $\%$ for accuracy, $89.8 \%$ for precision, $92.8 \%$ for recall, and 91.3 for the F1 score. Lastly, the VGG model attained the lowest measurements among other models by scoring $85.1 \%, 87.1 \%, 89.7 \%, 88.4 \%$ for the accuracy, precision, recall, and F1 score, respectively. Our outcomes proved that the aimed solution in scenario 2 is very powerful in handling the shortage of training data for skin cancer classification tasks. 
Table 1. The evaluation results from scenario 1

\begin{tabular}{ccccc}
\hline Model & Accuracy $(\%)$ & Precision $(\%)$ & Recall (\%) & F1 score (\%) \\
\hline VGG19 & 79.6 & 83.7 & 85.4 & 84.6 \\
GoogleNet & 81.2 & 84.4 & 87.5 & 85.9 \\
ResNet-50 & 84.0 & 86.6 & 89.3 & 87.9 \\
\hline
\end{tabular}

Table 2. The evaluation results from scenario 2

\begin{tabular}{ccccc}
\hline Model & Accuracy $(\%)$ & Precision $(\%)$ & Recall $(\%)$ & F1 score $(\%)$ \\
\hline VGG19 & 85.1 & 87.1 & 89.7 & 88.4 \\
GoogleNet & 88.8 & 89.8 & 92.8 & 91.3 \\
ResNet-50 & 93.7 & 95.7 & 94.6 & 95.1 \\
\hline
\end{tabular}

\section{CONCLUSION}

In this article, we presented a novel strategy of transfer learning to tackle the issue of shortage of training data in skin cancer classification tasks by turning the learned features of pre-trained models of the ImageNet. We trained the models on a large number of unlabelled skin cancer images. We then train them on a small number of labeled skin. This approach guaranteed that the models learned the relevant features of the skin cancer classification task. The results demonstrated that the aimed method is beneficial by performing an accuracy of $84 \%$ with ResNet50 when directly trained with a small number of labeled skin and $93.7 \%$ when trained with the proposed approach. This approach is suitable for any medical imaging task that has the issue of providing sufficient labeled images. We intend to apply the aimed strategy for other medical imaging applications. We also aim to use new type of transfer learning called same-domain transfer learning.

\section{REFERENCES}

[1] L. Alzubaidi et al., "Novel Transfer Learning Approach for Medical Imaging with Limited Labeled Data," Cancers, vol. 13, no. 7, 1590, 2021, doi: 10.3390/cancers13071590.

[2] K. H. M. Celebi, B. Uddin, H. Iyatomi, Y. Aslandogan, W. Stoecker and R. Moss, "A methodological approach to the classification of dermoscopy images," Comput. Med. Imag. Grap., vol. 31, no. 6, pp. 362-373, 2007, doi: 10.1016/j.compmedimag.2007.01.003.

[3] A. R. A. Ali and T. M. Deserno, "A systematic review of automated melanoma detection in dermatoscopic images and it's ground truth data," Proc. SPIE Med. Imag., vol. 8318, pp. 83181I-1-83181I-11, 2012, doi: $10.1117 / 12.912389$.

[4] N. Codella, J. Cai, M. Abedini, R. Garnavi, A. Halpern and J. R. Smith, "Deep Learning, Sparse Coding, and SVM for Melanoma Recognition in Dermoscopy Images," presented at the Proc. Med. Imag. Comput. Comput. Assist. Interv., 2015, doi: 10.1007/978-3-319-24888-2_15.

[5] D. Gutman et al., (2016, Skin Lesion Analysis toward Melanoma Detection: "A Challenge at the International Symposium on Biomedical Imaging (ISBI) 2016", hosted by the International Skin Imaging Collaboration (ISIC).

[6] L. Alzubaidi, O. Al-Shamma, M. A. Fadhel, L. Farhan, J. Zhang and Y. Duan, "Optimizing the performance of breast cancer classification by employing the same domain transfer learning from hybrid deep convolutional neural network model," Electronics, vol. 9, no. 3, p. 445, 2020, doi: 10.3390/electronics9030445.

[7] L. Alzubaidi, M. A. Fadhel, O. Al-Shamma, J. Zhang, J. Santamaría, Y. Duan, and S. R. Oleiwi, "Towards a better understanding of transfer learning for medical imaging: a case study," Applied Sciences, vol. 10, no. 13, p. 4523, 2020, doi: 10.3390/app10134523.

[8] R. I. Hasan, S. M. Yusuf, and L. Alzubaidi, "Review of the state of the art of deep learning for plant diseases: A broad analysis and discussion," Plants, vol. 9, no. 10, p. 1302, 2020, doi: 10.3390/plants9101302.

[9] L. Alzubaidi, M. A. Fadhel, O. Al-Shamma, J. Zhang and Y. Duan, "Deep learning models for classification of red blood cells in microscopy images to aid in sickle cell anemia diagnosis," Electronics, vol. 9, no. 3, p. 427, 2020, doi: 10.3390/electronics9030427.

[10] L. Alzubaidi, M. A. Fadhel, O. Al-Shamma, J. Zhang, J. Santamaría, and Y. Duan, "Robust application of new deep learning tools: an experimental study in medical imaging," Multimedia Tools and Applications, pp. 1-29, 2021, doi: 10.1007/s11042-021-10942-9.

[11] L. Alzubaidi, et al., "Review of deep learning: concepts, CNN architectures, challenges, applications, future directions," Journal of Big Data, vol. 8, no. 1, pp. 1-74, 2021, doi: 10.1186/s40537-021-00444-8.

[12] AG. Pacheco and Krohling R, "An attention-based mechanism to combine images and metadata in deep learning models applied to skin cancer classification," IEEE journal of biomedical and health informatics, Feb 26, 2021, doi: 10.1109/JBHI.2021.3062002.

[13] HW. Huang, B. W. Hsu, C. H. Lee and V. S. Tseng, "Development of a light-weight deep learning model for cloud applications and remote diagnosis of skin cancers," The Journal of Dermatology, Mar, vol. 48, no. 3, pp. 310-6, 2021, doi: 10.1111/1346-8138.15683.

[14] J. Deng, W. Dong, R. Socher, L. J. Li, L. Kai, and F.-F. Li, "ImageNet: A large-scale hierarchical image database," presented at the Proc. IEEE Conf. Comput. Vis. Pattern Recognit., 2009, doi: 10.1109/CVPR.2009.5206848. 
[15] J. Kawahara, A. BenTaieb, and G. Hamarneh, "Deep features to classify skin lesions," presented at the Proc. IEEE 13th Int. Symp. Biomed. Imag., 2016, doi: 10.1109/ISBI.2016.7493528.

[16] A. Menegola, M. Fornaciali, R. Pires, F. V. Bittencourt, S. Avila, and E.Valle, "Knowledge transfer for melanoma screening with deep learning," presented at the Proc. IEEE 14th Int. Symp. Biomed. Imag., 2017, doi: 10.1109/ISBI.2017.7950523.

[17] Y. Gong, L. Wang, R. Guo, and S. Lazebnik, "Multi-scale Orderless Pooling of Deep Convolutional Activation Features," presented at the Proc. 13th Eur. Conf. Comput. Vis., Cham, 2014, doi: 10.1007/978-3-319-10584-0_26.

[18] Howard, Andrew G. "Some improvements on deep convolutional neural network based image classification," arXiv preprint arXiv:1312.5402, 2013.

[19] Z. Hang, X. Jia, and D. Kristin, "Deep TEN: Texture Encoding Network," presented at the Proc. IEEE Conf. Comput. Vis. Pattern Recognit., 2017, doi: 10.1109/cvpr.2017.309.

[20] Y. Song, Q. Li, H. Huang, D. Feng, M. Chen, and W. Cai, "Low Dimensional Representation of Fisher Vectors for Microscopy Image Classification, "IEEE Trans. Med. Imag., vol. 36, no. 8, pp. 1636-1649, 2017, doi: 10.1109/TMI.2017.2687466.

[21] D. Yoo, S. Park, J.-Y. Lee, and I. So Kweon, "Multi-scale pyramid pooling for deep convolutional representation," presented at the Proc. IEEE Conf. Comput. Vis. Pattern Recognit. Workshops, 2015, doi: 10.1109/CVPRW.2015.7301274.

[22] Z. Yu, X. Jiang, T. Wang, and B. Lei, "Aggregating Deep Convolutional Features for Melanoma Recognition in Dermoscopy Images," presented at the Mach. Learn. Med. Imag., Cham, 2017, doi: 10.1007/978-3-319-67389-9_28.

[23] J. Yue-Hei Ng, F. Yang, and L. S. Davis, "Exploiting local features from deep networks for image retrieval," presented at the Proc. IEEE Conf. Comput. Vis. Pattern Recognit. Workshop, 2015, doi: 10.1109/cvprw.2015.7301272.

[24] M. Cimpoi, S. Maji, and A. Vedaldi, "Deep filter banks for texture recognition and segmentation," presented at the Proc. IEEE Conf. Comput. Vis. Pattern Recognit., 2015, doi: 10.1109/CVPR.2015.7299007.

[25] K. Korotkov and R. Garcia, "Computerized analysis of pigmented skin lesions: A review," Artif. Intell. Med., vol. 56, pp. 69-90, 2012, doi.org/10.1016/j.artmed.2012.08.002.

[26] H. Ganster, P. Pinz, R. Rohrer, E. Wildling, M. Binder, and H. Kittler, “Automated melanoma recognition," IEEE Trans. Med. Imag., vol. 20, no. 3, pp. 233-239, 2001, doi: 10.1109/42.918473.

[27] G. Capdehourat, A. Corez, A. Bazzano, R. Alonso, and P. Musé, "Toward a combined tool to assist dermatologists in melanoma detection from dermoscopic images of pigmented skin lesions," Pattern Recognit. Lett., vol. 32, no. 16, pp. 2187-2196, 2011, doi: 10.1016/j.patrec.2011.06.015

[28] F. Xie, H. Fan, L. Yang, Z. Jiang, R. Meng, and A. Bovik, "Melanoma Classification on Dermoscopy Images using a Neural Network Ensemble Model," IEEE Trans. Med. Imag., vol. 36, no. 3, pp. 1-1, 2016, doi: 10.1109/TMI.2016.2633551.

[29] L. Bi, J. Kim, E. Ahn, D. Feng, and M. Fulham, "Automatic melanoma detection via multi-scale lesion-biased representation and joint reverse classification," presented at the Proc. IEEE 13th Int. Symp. Biomed. Imag., 2016, doi: 10.1109/ISBI.2016.7493447.

[30] C. Barata, M. Ruela, M. Francisco, T. Mendonça, and J. S. Marques, "Two Systems for the Detection of Melanomas in Dermoscopy Images Using Texture and Color Features," IEEE Systems Journal, vol. 8, no. 3, pp. 965-979, 2014, doi: 10.1109/JSYST.2013.2271540.

[31] L. Alzubaidi, Fadhel, M. A., Oleiwi, S. R., Al-Shamma, O., and Zhang, J, "DFU_QUTNet: diabetic foot ulcer classification using novel deep convolutional neural network," Multimedia Tools and Applications, vol. 79, no. 21, pp. 15655-15677, 2020, doi: 10.1007/s11042-019-07820-w.

[32] A. A. Abbood, Q. M. Shallal, and M. A. Fadhel, "Automated brain tumor classification using various deep learning models: a comparative study,"Indonesian Journal of Electrical Engineering and Computer Science (IJEECS), vol. 22, no. 1, pp. 252-259, 2021, doi: 10.11591/ijeecs.v22.i1.pp252-259.

[33] L. Alzubaidi, R. I. Hasan, F. H. Awad, M. A. Fadhel, O. Alshamma, and J. Zhang, "Multi-class Breast Cancer Classification by a Novel Two-Branch Deep Convolutional Neural Network Architecture," In 2019 12th International Conference on Developments in eSystems Engineering (DeSE), pp. 268-273. IEEE, 2019, October, doi: 10.1109/DeSE.2019.00057.

[34] S. Demyanov, R. Chakravorty, M. Abedini, A. Halpern, and R. Garnavi, "Classification of dermoscopy patterns using deep convolutional neural networks," presented at the Proc. IEEE 13th Int. Symp. Biomed. Imag., 2016, doi: 10.1109/ISBI.2016.7493284.

[35] L. Yu, H. Chen, Q. Dou, J. Qin, and P. A. Heng, "Automated Melanoma Recognition in Dermoscopy Images via Very Deep Residual Networks," in IEEE Transactions on Medical Imaging, vol. 36, no. 4, ed, 2016, pp. 1-1, doi: 10.1109/TMI.2016.2642839.

[36] A. Esteva et al., "Dermatologist-level classification of skin cancer with deep neural networks," Nature, vol. 542, pp. 115-118, 2017, doi: 10.1038/nature21056.

[37] N. Codella, Q.-B. Nguyen, S. Pankanti, D. Gutman, B. Helba, A. Halpern, "Deep Learning Ensembles for Melanoma Recognition in Dermoscopy Images," vol. 61, pp. 4, 2016, doi: 10.1147/jrd.2017.2708299.

[38] Z. Yu et al., "Hybrid dermoscopy image classification framework based on deep convolutional neural network and Fisher vector," presented at the Proc. IEEE 14th Int. Symp. Biomed. Imag, 2017, doi: 10.1109/ISBI.2017.7950524.

[39] L. Alzubaidi, O. Al-Shamma, M. A. Fadhel, L. Farhan, and J. Zhang, "Classification of red blood cells in sickle cell anemia using deep convolutional neural network," In International Conference on Intelligent Systems Design and Applications, pp. 550-559. Springer, Cham, 2018, doi: 10.1007/978-3-030-16657-1_51. 
[40] M. A. Fadhel, O. Al-Shamma, S. R. Oleiwi, B. H. Taher, and L. Alzubaidi, "Real-time PCG diagnosis using FPGA," In International Conference on Intelligent Systems Design and Applications, pp. 518-529. Springer, Cham, 2018, doi: 10.1007/978-3-030-16657-1_48.

[41] O. Al-Shamma, M. A. Fadhel, R. A. Hameed, L. Alzubaidi, and J. Zhang, "Boosting convolutional neural networks performance based on FPGA accelerator," In International Conference on Intelligent Systems Design and Applications, pp. 509-517. Springer, Cham, 2018, doi: 10.1007/978-3-030-16657-1_47.

[42] The International Skin Imaging Collaboration | Challenges. Accessed: Dec. 1, 2020. [Online]. Available: https://www.isic-archive.com/\#!/topWithHeader/tightContentTop/challenges.

[43] SIIM-ISIC Melanoma Classification. https://www.kaggle.com/c/siim-isic-melanoma-classification. Accessed: 26 December 2020

[44] K. Simonyan, and A. Zisserman, "Very deep convolutional networks for large-scale image recognition," arXiv preprint arXiv:1409.1556. 2014

[45] C. Szegedy, W. Liu, Y. Jia, P. Sermanet, S. Reed, D. Anguelov and A. Rabinovich, "Going deeper with convolutions," In: Proceedings of the IEEE conference on computer vision and pattern recognition, pp. 1-9, 2015, doi: 10.1109/CVPR.2015.7298594.

[46] K. He, X. Zhang, S. Ren and J. Sun, "Deep residual learning for image recognition," In Proceedings of the IEEE conference on computer vision and pattern recognition, pp. 770-778, 2016, doi: 10.1109/CVPR.2016.90.

[47] O. Russakovsky, J. Deng, H. Su, J. Krause, S. Satheesh, S. Ma and AC. Berg, "ImageNet large scale visual recognition challenge," Int J Comput Vis, vol. 115, no. 3, pp. 211-252, 2015, doi: 10.1007/s11263-015-0816-y.

[48] H. Al-Yassin, J. I. Mousa, M. A. Fadhel, O. Al-Shamma, and L. Alzubaidi, "Statistical accuracy analysis of different detecting algorithms for surveillance system in smart city," Indonesian Journal of Electrical Engineering and Computer Science (IJEECS), vol. 18, no. 2, pp. 979-986, 2020, doi: 10.11591/ijeecs.v18.i2.pp979-986.

[49] A. Al-Sabaawi, H. M. Ibrahim, Z. M. Arkah, M. Al-Amidie, and L. Alzubaidi, "Amended Convolutional Neural Network with Global Average Pooling for Image Classification," In International Conference on Intelligent Systems Design and Applications, pp. 171-180, Springer, Cham, 2020, doi: 10.1007/978-3-030-71187-0_16.

[50] L. Alzubaidi, O. Al-Shamma, M. A. Fadhel, Z. M. Arkah and F. H. Awad, "A deep convolutional neural network model for multi-class fruits classification," In International Conference on Intelligent Systems Design and Applications, pp. 90-99. Springer, Cham, 2019, December, doi: 10.1007/978-3-030-49342-4_9.

[51] L. Alzubaidi, A. A. Abbood, M. A. Fadhel, O. Al-Shamma and J. Zhang, "Comparison of hybrid convolutional neural networks models for diabetic foot ulcer classification," Journal of Engineering Science and Technology, vol. 16, no. 3, pp. 2001-2017. 2021

[52] O. Alshamma, F. H. Awad, L. Alzubaidi, M. A. Fadhel, Z. M. Arkah and L. Farhan, "Employment of Multiclassifier and Multi-domain Features for PCG Recognition," In 2019 12th International Conference on Developments in eSystems Engineering (DeSE), pp. 321-325. IEEE, 2019, doi: 10.1109/DeSE.2019.00066.

\section{BIOGRAPHIES OF AUTHORS}

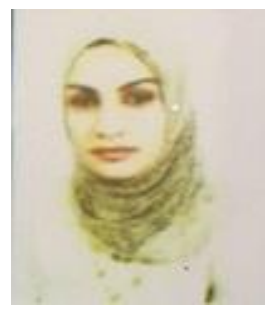

Zinah Mohsin Arkah M.Sc. in Computer Science and Technology from Southwest Jiaotong University, China -2017. Currently, I'm working as a lecture at the University of information technology and Communication. My research area interests are Deep Learning, Machine learning, data mining, big data, Image processing and Digital Image Processing.

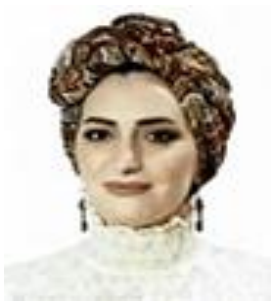

Dalya S. Al-Dulaimi Lecture at Universiyu of Information Technology and Communication Medical Informatics College. B.Sc. degree in Computer Science/ Baghdad University (Iraq). M.Sc. degree in Information Technology/ Çankaya University (Turkey). My specialized field in Medical Information Technology.

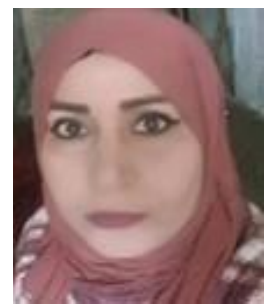

Ahlam Rashid Khekan B.Sc. degree in computer science /Al-Mustansirya University (Iraq), M.Sc. degree in computer science /Al-Mustansirya University (Iraq). My specialize Artificial Intelligence 